\title{
Proceedings of the meeting of the Association of British Neurologists, Glasgow, 6-8 April 1989
}

ATRIAL FIBRILLATION AND STROKE: THE OXFORDSHIRE COMMUNITY STROKE PROJECT PAG Sandercock, J Bamford, M Dennis, J Burn, CP Warlow, J Slattery, L Jones. Western General Hospital, Edinburgh

We examined data on the clinical features and prognosis of 675 patients with first-ever stroke and 184 patients with Transient Ischaemic Attack (TIA) in the Oxfordshire Community Stroke Project. The prevalence of atrial fibrillation in patients with cerebral infarction was $17 \%$, primary intracerebral haemorrhage $10 \%$, subarachnoid haemorrhage $0 \%$ and TIA $14 \%$. In subtypes of cerebral infarction the prevalence was total anterior cerebral infarction $31 \%$, partial anterior cerebral infarction $17 \%$, lacunar infarction $11 \%$, and posterior circulation infarction $13 \%$. In almost all pathological groups 30 day case fatality rates were 2-3 fold higher in patients in atrial fibrillation compared to patients in sinus rhythm. However, amongst patients who survived at least 30 days from their first stroke, the risk of recurrent stroke or recurrent stroke/vascular death was similar in patients in atrial fibrillation to patients in sinus rhythm. These data suggest that not all strokes in patients with atrial fibrillation are due to embolism from the heart, and survivors of first stroke in atrial fibrillation do not have a large excess risk of recurrent stroke. These data underline the need for randomised trials of the value of anticoagulants in patients with stroke and atrial fibrillation, and the need to perform CT scanning before starting anticoagulant therapy.

\section{IMPACT OF CONTRALATERAL INTERNAL} CAROTID ARTERY DISEASE ON OUTCOME OF SYMPTOMATIC INTERNAL CAROTID OCCLUSION JPH Wade, W Taylor, V Hachinski, HJM Barnett. Charing Cross Hospital, London

The contralateral internal carotid artery (ICA) becomes a major source of collateral supply in patients with unilateral ICA occlusion. Physiological studies have shown diminished haemodynamic reserve when the contralateral artery becomes severely stenosed or occluded. Contralateral ICA sten- osis is amenable to endarterectomy: so does the presence/degree of contralateral ICA stenosis adversely influence outcome in unilateral ICA occlusion?

In the EC/IC Bypass Study, 339 patients with symptomatic (TIA and/or small completed stroke) ICA occlusion were randomised to the medical group. Of these, 60 had no contralateral disease, 160 had mild $(0-30 \%)$ stenosis, 92 had moderate $(30$ $70 \%)$ stenosis and 27 had severe contralateral (70-99\%) stenosis. Forty percent of those with severe contralateral ICA stenosis suffered a stroke during follow-up (mean duration 60 months) whereas $30 \%$ of those with lesser degrees of stenosis had a stroke. The differences were not statistically significant. When patients with none/ moderate degrees of contralateral stenosis were lumped together $(n=220)$ and compared against those with moderate to severe contralateral stenosis $(n=119)$ stroke rates during follow-up were similar.

These results suggest that contralateral IC endarterectomy should not necessarily be undertaken in patients with symptomatic IC occlusion and extensive contralateral disease.

FEASIBILITY OF PERCUTANEOUS

TRANSLUMINAL ANGIOPLASTY FOR INTERNAL CAROTID ARTERY STENOSIS

MM Brown, P Butler, J Gibbs, M Swash, J Waterson. The London Hospital, London

Percutaneous transluminal angioplasty (PTA) was attempted in seven patients with internal carotid artery stenosis. All had recurrent neurological symptoms considered haemodynamic in origin. Six had occlusion of the contralateral internal carotid artery. Cerebral blood flow studies confirmed diminished cerebrovascular reserve. In four patients PTA was successfully carried out with apparent benefit. In two patients PTA could not be performed because of technical difficulty crossing the stenosis and in one PTA was abandoned because there was atheromatous ulceration of the carotid artery. One patient in whom PTA could not be completed developed transient aphasia during cannulation of the carotid artery, and one patient in whom PTA was successful developed a transient monoparesis during the procedure. Both these haemodynamic complications recovered within ten minutes. No persiste complications occurred.

Carotid angioplasty is technically feasible and may prove useful in the management $8 \mathrm{f}$ stenotic carotid disease associated wi haemodynamic stroke. PTA is a technically simple procedure requiring only brief hosp italisation. Its general application patients with thromboembolic stroke wil depend on the risk benefit ratio compared to carotid endarterectomy or convention: medical treatment.

INTRACRANIAL HAEMODYNAMICS IN TAKAYASU'S ARTERITIS

DG Grosset, J Patterson, I Bone. Sourhę General Hospital, Glasgow

Intracranial haemodynamics in two km cases of Takayasu's arteritis were stufied. Angiography in a 48 year old lady shows right innominate artery occlusion and $1 \& \mathrm{ft}$ subclavian stenosis. Single photon emission computed tomography (SPECT) showed reduced right sided cerebral blood flow in the territories supplied by the anterior and middle cerebral arteries. Transcranial Dofpler Sonography (TCD) found left to right shunting within the circle of Willis, anci left to right vertebro-vertebral shunt due $\$$ subclavian steal.

By contrast, a 35 year old lady with right subclavian occlusion and tight stenosis of the right common carotid artery, showed normal results on both SPECT while TC showed an extended subclavian steal with carotid-basilar-vertebral shunting.

It is concluded that the basic structural information provided by cerebral angioge phy, in such cases of multifocal vessel disease, requires supplementation by cerebFal blood flow studies. SPECT demonstrates end-result, in terms of cerebral perfusion. and TCD provides unique functional infoomation, in evaluating intracrana haemodynamics in such patients. 
VERTIGO IN VERTEBRO-BASILAR

INSUFFICIENCY

LM Luxon. The National Hospitals for Nervous Diseases, London

Elderly patients with recurrent episodes of vertigo are not uncommonly diagnosed as suffering from vertebro-basilar insufficiency (VBI). Justification for this derives from a well-documented series of patients with VBI in whom vertigo has been demonstrated to be the first and most frequent symptom. However, VBI represents only one pathophysiological mechanism associated with diffuse cerebro-vascular atherosclerosis, and the posterior circulation supplies both peripheral and central vestibular pathways. To characterise vestibular symptoms associated with VBI, 40 patients (mean age 61 years), in whom this diagnosis was made, using strict clinical criteria, were studied retrospectively; $75 \%$ suffered vertigo, but only $10 \%$ had episodes of isolated vertigo. Twenty five percent suffered dizziness and/ or unsteadiness and $7 \%$ had no symptom of disequilibrium. Vestibular investigations revealed abnormalities (as judged against normative data) in $90 \%$. Six patients had evidence of a peripheral (labryinthine/ eighth nerve) pathology only; eight patients had central vestibular (brainstem/ cerebellar) pathology, and the remaining six had combined pathology. All patients with a peripheral abnormality had suffered vertigo. In no two patients were identical abnormalities documented and no characteristic pattern of findings emerged. Isolated vertigo is an unusual manifestation of VBI and other vestibular pathologies should be excluded. However, vertigo is the commonest symptom in VBI, as a result of ischaemia of both peripheral and central vestibular pathways.

MAGNETIC STIMULATION AS A QUANTIFIER OF MOTOR DISABILITY

RH Kandler, JA Jarrett, EJW Gumpert, GAB Davies-Jones, GS Venables, HJ Sagar. Royal Hallamshire Hospital, Sheffield

Magnetic stimulation was used to measure motor conduction time (MCT) in pathways between head and neck in 27 normal subjects, 89 patients with definite multiple sclerosis (MS), 37 with Parkinson's disease (PD) and 14 with acute cerebrovascular accident (CVA).

Abnormal MCTs were found in $56 \%$ of MS patients: $64 \%$ of those with and $8 \%$ of those without pyramidal signs. There was a significant correlation between MCT and
Kurtzke grading for pyramidal dysfunction $\left(r_{s}=0.64 ; p<0.01\right)$. MCTs, which were abnormal in a group of 18 patients in acute relapse from MS, were re-measured after steroid treatment. The mean value shortened in patients who improved clinically $(17.7$ to $15.3 \mathrm{msec} ; \mathrm{p}<0.01)$ but not in those who remained clinically unchanged (14.4 to $14.4 \mathrm{msec})$.

PD patients had shorter MCTs (mean 7.9 ms, SD 1.4) and higher amplitude responses from stimulation at the head (mean $1.7 \mathrm{mV}$, SD 1.4) than normal subjects (mean MCT $9.9 \mathrm{~ms}$, SD 1.3; mean amplitude $0.9 \mathrm{mV}, \mathrm{SD}$ $1.0),(p<0.01)$.

The seven patients with CVA who had made an incomplete recovery by the fourth week after stroke had absent or small motor responses to cortical stimulation on the fourth day after the ictus.

Magnetic stimulation promises to be useful in quantifying both pyramidal and extra pyramidal dysfunction; it may also be a useful prognostic indicator for recovery in acute stroke.

BRAIN IMPLANTS IN MAN DO NOT BREAK DOWN THE BLOOD-BRAIN BARRIER TO DOPAMINE AND DOMPERIDONE

CG Clough, ER Hitchock, RC Hughes. Midland Centre for Neurosurgery and Neurology, Birmingham

Neocortical implants in the rat brain lack a blood-brain barrier (BBB) to macromolecules. If this occurred following implantation in man it could explain the early improvement since neurotransmitters such as dopamine which would not normally cross the BBB might gain access to striatal dopamine receptors; alternatively these areas may be open to cerebrospinal fluid. Eight patients (six males, two females, age 41-67 years, mean 56.4 years) have undergone stereotactic implantation of foetal mesencephalon into the head of the right caudate nucleus and one patient (male, aged 46 years) had autologous adrenal medulla implanted into the right caudate nucleus and attached to the ventricular wall. All patients had dopamine infusions ( $<2000 \mathrm{mgm} /$ minute) followed by apomorphine (1.5 mg sc) and domperidone (20 mg per oral). Patients were assessed every five minutes by a standard Parkinsonian rating scale. No patients showed a response to dopamine but all patients responded to apomorphine and domperidone. The test was conducted pre-operatively and five days post-operatively in four patients and at one, two, three, four months and one year post-operatively in the other patients.
We have demonstrated that dopamine and domperidone do not have access to striatal dopaminergic post-synaptic receptors.

GADOLINIUM ENHANCEMENT IS A MARKER FOR BLOOD-BRAIN BARRIER BREAKDOWN IN INFLAMMATORY DEMYELINATION

CP Hawkins, $P$ Munro, J Kesselring, P Tofts, EPGH du Boulay, DN Landon, WI McDonald. Institute of Neurology, London

Multiple sclerosis (MS) lesions frequently enhance with Gadolinium-DTPA (GdDTPA) at MRI. The histological basis for this is of considerable interest because of its implications for understanding the evolution of the plaque and its potential use in monitoring treatment. Because of the histological similarities between the lesions of MS and chronic relapsing experimental allergic encephalomyelitis (CREAE) in guinea-pigs, we have undertaken a serial study of the evolution of BBB changes in EAE and related them to the histological findings.

Forty strain 13 and 10 Hartley guinea-pigs with EAE were studied. Gd-DTPA enhancement occurred only in areas of perivas- $T$ cular inflammation and corresponded with $\bar{P}$ sites of BBB breakdown to traditional mark $\mathbb{D}$ ers (horseradish peroxide, lanthanumb nitrate). Enhancement also occurred with Gd-IgG conjugates indicating increased per meability to large molecules. Duration of Gd-DTPA enhancement for individuap lesions in CREAE varied from seven days to more than five weeks in contrast to acute EAE, in which enhancement lasted less than seven days.

From the similarities in the histological features and pattern of enhancement of CREAE and MS, we conclude that GdDTPA is a marker for inflammation and therefore provides a marker for disease activity which will be useful in monitoring therapy.

THE NATURAL HISTORY OF THE MULTIPLE SCLEROSIS LESION

AG Kermode, PS Tofts, $P$ Rudge, A Thompson, EPGH du Boulay, BE Kendall, IF Moseley, WI McDonald. Institute of Neurology, London

We have studied the natural history of the multiple sclerosis plaque in 11 patients with clinically definite multiple sclerosis by serial MRI and quantification of the blood brain barrier using Gd-DTPA. Breakdown in the blood brain barrier is seen in all new lesions, and in dynamic scans peak enhancement was reached almost as rapidly as in the choroid plexus where there is no blood-tissue barrier. 
There is a progressive decrease in permeability in the following weeks. Many chronic lesions show enhancement at four minutes. With rapid scanning new lesions enhance uniformly whereas lesions older than one month often appear as rings, which fill by 20 minutes. After five hours there is centrifugal diffusion of gadolinium. In some such lesions the diameter of the ring increases over a matter of weeks. From a comparison of the in-vivo data, with post mortem findings and data from chronic relapsing experimental allergic encephalomyelitis, we conclude that a constant early event in the development of the new plaque is a breakdown in the blood brain barrier; the "rings" indicate renewed activity at the periphery of old lesions which increase in size centrifugally. These observations are potentially useful in monitoring treatment.

\section{MULTIPLE SCLEROSIS IN THE SOUTHAMPTON} AND SW HAMPSHIRE HEALTH AUTHORITY M Roberts, D McLellan, J Martin, S McIntosh, A Spackman. Southampton General Hospital, Southampton

The Southampton Multiple Sclerosis Survey is a population based prevalence study in the Southampton and South-West Hampshire Health Authority (1986 population 417,000 ). This has included interviews with relatives and psychometric assessment, aspects neglected in previous epidemiological studies. A provisional list of 795 people thought to have MS yielded 411 people with MS who were usually resident in the Health Authority on the 1st January 1987. General Practitioners and the local MS Society branch were the most fruitful sources. Diagnosis was by review of case-notes plus personal interview in $305(74 \%)$. The prevalence was $99 / 100,000(95 \%$ CI $89-109)$ in all Allison and Millar categories and 92/100,000 (95\% CI 83-101) excluding possibles. The mean age was 48.6 years, mean age of onset 32.6 years, and mean duration 15.7 years. The sex ratio was $2 \cdot 1$ females to males. Thirty one percent of those interviewed had Kurtzke Expanded Disability Status Scale scores of 7.0 or above. One third had regular urinary incontinence. This study accords with the recent South Glamorgan and Sutton studies that the prevalence of MS in the south of the United Kingdom is higher than usually quoted.

CLUSTERING OF BIRTHDATES OF PATIENTS WITH MULTIPLE SCLEROSIS AND PROBABLE PARKINSON'S DISEASE IN GRAMPIAN REGION MC Pitt, JEC Hern, AM Carrick, DF Kerridge. University of Aberdeen, Aberdeen
It has long been suspected that events in early childhood can influence the chances of developing multiple sclerosis (MS). Over many years, a register of patients with MS has been built up for Grampian Region. It now contains details of 1645 patients. The pattern of their birthdates did not appear to be random. After excluding 628 not born in Grampian, or whose birthplace was not recorded, the pattern of clustering was even more clear. Among other possibilities, such clustering could arise from exposure to some infection around the time of birth. We have not been able to find a correlation with any particular infection.

It has been recently suggested that the risk of developing Parkinson's Disease (PD) may be related to intra-uterine exposure to influenza. For another study, we have collected the names and birthdates of all 468 patients in Grampian for whom preparations containing L-dopa were dispensed in two summer months of 1986 . It is likely that most have PD. Their birthdates showed clustering, though at times different from those for MS and from most of the clusters of influenza deaths.

CHROMATIC VISUAL EVOKED POTENTIALS AND PSYCHOPHYSICAL FINDINGS IN MULTIPLE SCLEROSIS

MHA Russell, IJ Murray, RA Metcalfe, JJ Kulikowski. Manchester Royal Infirmary, Manchester

In human vision separate neural pathways exist for the processing of luminance and colour information. In multiple sclerosis, selective impairment of one or the other of these pathways may occur. An evoked potential technique has been developed in which chromatic pathways can be selectively activated by the onset of isoluminant redgreen bars. Achromatic pathways are similarly tested using luminance modulated patterns.

In this study 32 patients with multiple sclerosis were investigated using chromatic and achromatic VEPs, spectral sensitivity (SS) and critical flicker fusion frequency (CFF). SS monitors the activity of the colour opponent mechanisms if the test spot is presented at $1 \mathrm{~Hz}$ and the luminance system at $25 \mathrm{~Hz}$. The results of the psychophysical assessments (SS and CFF) were compared with the VEPs.

SS at $1 \mathrm{~Hz}$ was equally reduced for short, medium and long wavelengths. Depressed 25 $\mathrm{Hz}$ SS was correlated with abnormal latencies of both chromatic and achromatic VEPs. Achromatic VEP amplitudes were not correlated and $1 \mathrm{~Hz}$ SS. Finally, reduced amplitude of the chromatic VEP occurred those patients with reduced $1 \mathrm{~Hz}$ SS but $d \mathrm{dd}$ not correlate with reduced $25 \mathrm{~Hz}$ SS. Thōs the chromatic VEP can be used as an obje tive measure of colour vision in multiple sclerosis.

DEFICIENCIES IN ACHR ANTIBODY MEASUREMENT IN MYASTHENIA GRAVIS CE Clarke, DI Shepherd, GM Yuill, J Smaje. North Manchester General Hospita Manchester

The records were reviewed of the 86 cases Myasthenia Gravis (MG) presenting to the department over a nine year period. Of the patients alive at 1.8 .1988 , the Tensilon test was positive in $55(81 \%)$ of the 69 in which result was recorded. Those with ocular dis ease were less often positive $(59 \%)$ than those with generalised MG (89\% Repetitive stimulation was abnormal in 2 $(62 \%)$ of the 34 examined with generalise MG but in none of the 10 with ocular disease. Single fibre EMG of extensor digitorum communis was abnormal in nine $(82 \%)$ of $\longleftrightarrow$ with generalised $M G$ and in one $(17 \%$ seven with ocular MG. Striated ma antibodies were found in eight $(24 \%)$ oftip 33 patients tested, thymomas were presegt two of the three positive cases who underwent thymectomy. Only $20(36 \%)$ of the 56 patients examined for acetylcholine receptof (AChR) antibodies proved positive; $12 \%$ those with ocular MG were positive and $\frac{69}{4}$ of those with generalised MG.

These findings are largely in accord $\overrightarrow{\bar{W}} \overrightarrow{\mathrm{T}}$ those previously recorded. However, othe workers report a positive AChR antibod test in $66-93 \%$ of patients using anti-huma IgG anti-sera to precipitate the AchR antibody complex. The low sensitivity in the present study may have arisen from the use of Staphylococcal Protein A to precipitate the complex in our regional immunolog laboratory. This may not bind to IgG sub class 3 and may therefore fail to recognise the antibody in some patients.

\section{ACHR-SPECIFIC T LYMPHOCYTES IN} INDIVIDUALS WITHOUT MYASTHENIA GRAVIS $\frac{7}{0}$ N Sommer, G Harcourt, N Willcois D Beeson, J Newsom-Davis. Institute $\vec{\theta}$ Molecular Medicine, Oxford

We have studied $\mathrm{T}$ cell responses to Torped acetylcholine receptor (AChR) in myass thenia gravis (MG) patients and controls Patients' thymus and thymoma responded in more than $60 \%$ of cases $(<10 \%$ in control $\$$ and peripheral blood lymphocytes (PBL) if about $20 \%$. However, PBL from two of 28 
non-MG individuals responded significantly on several occasions, although the responses varied considerably over a period of 20 months.

Long term $\mathrm{T}$ cell lines to AChR could be raised from these two non-MG individuals and showed greatly increased antigen specificity up to 800 times the initial stimulation. All lines from controls as well as myasthenic patients had the phenotype of helper $\mathrm{T}$ cells $\left(\mathrm{CD}^{+} \mathrm{CD8}^{-}\right)$and were restricted by HLA-DR. Differences in fine specificity were found between patients and controls. One epitope on the (human) AChR alpha subunit was recognised by fresh PBL and by cloned cell lines from non-MG individuals, whereas $M G$ patients responded to at least two additional epitopes.

We conclude that AChR-specific T lymphocytes may be found in some healthy individuals, possibly cross-reacting with an environmental antigen. Their failure to induce myasthenia gravis may reflect differences in $T$ cell fine specificity or the presence of regulatory mechanisms.

IMPAIRED TRANSMITTER RELEASE IN A NEW CONGENITAL MYASTHENIC SYNDROME WITH A PAUCITY OF POST-SYNAPTIC FOLDS

PRW Fawcett, CR Slater, TJ Walls, D Gardner-Medwin. Newcastle General Hospital, Newcastle upon Tyne

A 19-year old man who presented at age 3 with continuous but variable weakness of the limbs became unable to walk by age 13 .

Neurophysiological studies at age 13 showed a $44 \%$ decrement at $3 \mathrm{~Hz}$ in deltoid and markedly increased single fibre EMG jitter and impulse blocking in extensor digiti communis. Serum anti-AChR antibody was absent. Treatment with pyridostigmine slightly improved strength and reduced fatiguability. At age 19, decrements of up to $63 \%$ were seen in ADM with repetitive stimulation at $1-20 \mathrm{~Hz}$, and post-activation facilitation $(6-30 \%)$ and exhaustion were present.

Intracellular recordings made in vitro from a motor-point biopsy of vastus lateralis showed normal sized miniature end plate potentials, but the number of quanta released from each nerve terminal at $1 \mathrm{~Hz}$ stimulation was only $24 \%$ of that in control muscles. Guanidine ( $5 \mathrm{mM}$ ) nearly doubled transmitter output. The indication of a major pre-synaptic defect was supported by stimulation single fibre EMG jitter studies showing reduced jitter at higher rates of stimulation. In the electron-microscope, neuro-muscular junctions consisted of presynaptic boutons of normal size and appearance associated with a post-synaptic membrane containing only $25 \%$ of the normal amount of folding.

Treatment with combined 3.4 diaminopyridine ( $20 \mathrm{mg}$ qds) and pyridostigmine (90 mg, three hourly) has resulted in almost complete resolution of the muscular weakness.

SINGLE PHOTON EMISSION COMPUTED TOMOGRAPHY IN ARTERIOVENOUS MALFORMATIONS

R Duncan, R Bullock, I Bone, J Patterson. Southern General Hospital, Glasgow

Shunting associated with arteriovenous malformations (AVM) may cause reduced perfusion in areas remote from the lesion itself. The precise extent of this has hitherto not been known. We present the results of single photon emission computed tomography (SPECT) in six patients with AVM, two of which were of the Sturge-Weber type.

In both cases of Sturge-Weber syndrome the extent of hypoperfusion was not only greater than the radiological extent of the AVM, but affected the opposite hemisphere and the territories of both middle and cerebral arteries. In one AVM the whole hemisphere was hypoperfused, while in two other cases hypoperfusion was minimal, despite the fact that one of these was large. In one case SPECT was carried out pre and post operatively, showing no change in the extent of hypoperfusion. One post operative scan showed marked hyperperfusion on the side of the AVM in a patient who developed hemiparesis. There is evidence that reperfusion of areas affected by steal may lead to improved function in areas remote from the AVM, but that overperfusion may cause neurological damage. Our findings show that SPECT can image perfusion abnormalities associated with AVM, and may well have a role in its pre and post operative assessment. Further study is warranted to establish this.

SPATIAL CONTRAST SENSITIVITY AND VISUAL ILLUSIONS IN CLASSICAL MIGRAINE:

CORRELATIONS WITH LATERALIZATION OF AURA

NM Khalil, NJ Legg. Hammersmith Hospital, London

Thirty seven patients with classical migraine and 30 control subjects were studied. Spatial contrast sensitivity was measured using Arden sinusoidal gratings, and by unilateral presentation of brief low-contrast gratings on a monochrome display monitor. The perception of visual illusions was assessed with the subjects looking at high-contrast static gratings of specific spatial frequency.

Subjects with migraine had poorer contrast sensitivity than controls, and this sensitivity was significantly lower in the visual field in which their visual aura was experienced. Illusions in response to high-contrast gratings were more common in migraine subjects than in controls, and were greater on the side of the aura. A long duration of migraine reduced contrast sensitivity, but not the illusions.

This suggests that in some migraine subjects there is a constitutional asymmetry of the visual system, which results in asymmetry of spatial contrast perception, and also the asymmetry of their visual auras. Thus these findings support a primary neural dysfunction in a classical migraine attack, and secondary vascular effects may cause long term deterioration in visual function as a result of repeated transient ischaemia.

NEUROPHYSIOLOGICAL ASSESSMENT CAN PROVIDE INDICATORS FOR LONG TERM PROGNOSIS IN THE GUILLAIN-BARRÉ SYNDROME

GA Jamal, JP Ballantyne. Southern General Hospital, Glasgow

Serial clinical and neurophysiological studies from time of onset for 18 months were performed on 20 patients with GuillainBarré syndrome to identify predictive indices and the optimum time for such an assessment. Patients were examined at one and/or three weeks after onset and thereafter at one, two, four, six, 12 and 18 months. Neurophysiological studies included quantitative EMG, sensory and motor conductions and late response studies, direct facial nerve conduction and blink reflexes and quantitative thermal and vibration perception thresholds.

Spontaneous activity on EMG was maximal in most patients at one month but in some at two months. The amount of spontaneous activity during such periods was closely related to the clinical improvement in power of the limb or facial muscles. Similar correlation was also found with the size of the compound muscle action potential at one month. The mean time for normalisation of abnormal thermal and vibration perception thresholds was also highly correlated with the severity of EMG and compound muscle action potential changes in the extremity muscles.

The findings suggest that neurophysiological evidence of severe axonal disruption is paralleled by slow and incomplete clinical recovery whereas predominant 
demyelination correlates with a relatively rapid and more complete recovery. Assessment of these prognostic indicators is best performed at one month and not later than two months following onset of the illness.

LATERLISING TEMPORAL LOBE ABNORMALITIES WITH SPECT AND MEMORY TESTS

R Gillham, R Duncan, J Patterson, I Bone. Southern General Hospital

Neurophysiological assessment is used routinely in screening potential candidates for temporal lobectomy. It is generally assumed that in left hemisphere dominant individuals verbal memory impairment is associated with left temporal dysfunction and non-verbal memory impairment with right. Single photon emission computed tomography (SPECT) and psychometric memory testing were used independently to lateralise temporal lobe abnormalities in 42 patients with temporal lobe epilepsy, to establish the degree of correspondence between the two methods.

Sixteen patients showed normal perfusion in both temporal lobes on SPECT. Five of these had verbal memory impairment, five non-verbal memory impairment and two both.

SPECT showed left temporal hypoperfusion in twelve patients. Of these six had verbal memory impairment, two non-verbal memory impairment, three both and one neither.

SPECT showed right temporal hypoperfusion in 14 patients. Nine of these had nonverbal memory impairment, one verbal memory impairment, three both and one neither.

The correspondence between SPECT localisation and specific memory impairment is imprecise, generally because more patients show neuropsychological abnormalities than would be predicted from SPECT. Patients with earlier age of onset and high total seizure count were more likely to show impairment. Early age of onset may be associated with mixed dominance and anomalous laterality. These significant factors tend to swamp subtle verbal/non-verbal differences.

\section{QUALITY OF LIFE FOLLOWING}

MICROVASCULAR DECOMPRESSION FOR

INTRACTABLE NEURALGIA: A FOUR YEAR

FOLLOW-UP STUDY

J Zakrewska, J Wade. Eastman Dental Hospital, London

The short-term results following microvascular decompression (MVD) for trigeminal neuralgia are good but to date long term follow-up data are limited. Sixty-four consecutive patients (mean age 54 years) who were treated by MVD because of failed medical treatment between 1978-1987 were studied. All were sent questionnaires and invited to attend for follow-up.

Six patients had died of unrelated causes; of the 58 potential responders, 54 returned their questionnaires $(93 \%), 41$ of whom also attended for follow-up. The mean duration of follow-up was 62 months (range 18-141). Fifty of the 64 subjects were pain free. Five had transient pain and nine suffered significant recurrence. One had a mild depression and nine were anxious, whereas these symptoms occurred in over half of a comparative pre-operative group. Forty-two subjects would undergo repeat surgery if their pain recurred. Neurological examination in 41 subjects revealed minor sensory signs in four and unilateral hearing loss in a further three subjects.

MVD provides good pain relief with minimal morbidity and results in a marked recovery in the quality of life.

LOCALISATION OF DYSTROPHIN IN HUMAN

MUSCLE BY GOLD IMMUNOLABELLING USING A MONOCLONAL ANTIBODY

MJ Cullen, J Walsh, LVB Nicholson, JB Harris. Newcastle General Hospital, Newcastle upon Tyne

We have used a monoclonal antibody to a fusion protein containing part of the dystrophin molecule to localise dystrophin at the ultrastructural level in cryosections of human skeletal muscle. After incubation with the primary anti-dystrophin antibody the sections were incubated with a secondary gold-conjugated goat anti-mouse antibody. In six non-dystrophic cases there was distinctive gold labelling located at the periphery of the fibres, close to the plasma membrane. In four cases of Duchenne muscular dystrophy there was no labelling.

The gold particles were concentrated in the outer $100 \mathrm{~nm}$ of the fibres (density = 27.08 per $\mu \mathrm{m}^{2}$ compared with 0.55 per $\mu \mathrm{m}^{2}$ in the rest of the fibre). The mean distance from the centre of the $5 \mathrm{~nm}$ gold particle to the cytoplasmic face of the plasma membrane was $15.8 \mathrm{~nm}$, and $94 \%$ of the label lay within $30 \mathrm{~nm}$ either side of this point. Measurement along the plasma membrane of the distance between "nearest neighbour" gold particles gave a unimodal negatively skewed distribution with a mode at $100 \mathrm{~nm}$.

These results support a model in which dystrophin normally forms a cytoskeletal meshwork close to the cytoplasmic surface of the plasma membrane of the muscle fibres.
DYSTROPHIN-POSITIVE FIBRES MAY BE MORE $\bar{Z}$ COMMON IN DUCHENNE PATIENTS THAN FIRS THOUGHT

LVB Nicholson, CR Slater, MA Johns@̋, JB Harris. Newcastle General Hospita, Newcastle upon Tyne

Studies on the phenotypic expression dystrophin in muscle have suggested thit Becker patients might have genetic deletio which permitted the production of a smalier "semi-functional" form of dystrophin, whine deletions in Duchenne (DMD) patients resulted in translation reading frame shifs and the absence of a protein product. $\overline{\bar{S}}$

Our results indicate that this is an ove simplification and that many DMD patiefts are able to make dystrophin-like proteinô, some of their muscle fibres.

We have raised monoclonal antibodies. $\overrightarrow{l_{0}}$ dystrophin using the Hoffman $30 \mathrm{kD}$ fusien protein and shown that these antibodies specific and do not cross-react with protein in $m d x$ mouse muscle. Prob g Western blots of human muscle from normial individuals with these antibodies reveale ${ }^{2}$ reproducible pattern of bands. Blots frem muscle of patients showed that at least fointe bands were visible in six of the first DMD biopsies tested. Immunocytochepicical analysis of frozen sections from these Giopsies showed labelling in isolated fibres or parts of fibres in eight of the nine sampless examined. Since all of the DMD pagiegts have gene deletions (7) and/or a history of disease (5) it is unlikelyêt that somatic reversion or mosaicism cause tbe rare dystrophin-positive fibres.

MOTOR NEURON DISEASE (MND) SERUM INCREASES THE TRH CONTENT OF CULTURED FETAL RAT BRAIN STEM CELLS MD Lewis, INF McQueen, MF Scanl\&̈. University of Wales College of Medicie, Cardiff

Thyrotrophin-releasing hormone (TR苗) producing neurons project from the median raphe nucleus of the brain stem to the cranial nerve motor nuclei and ventral spinal corfd where TRH may exert a trophic influence 8 n lower motor neuron (LMN) functiog. Therefore we studied the effects of sera from patients with motor neuron disease (MND) on TRH production in vitro.

Fetal rat brain stem cells were cultured for eight days and then treated with MNB serum $(30 \%$ in culture medium) for foyr days. Cells were then extracted and TRH measured using a sensitive and specific radi immunoassay subsequent to high pressure liquid chromotography purification of the cell extract. Within each experiment cultutss 
were treated with age and sex matched control sera. TRH content of MND seratreated cells $(\mathrm{n}=19$ patients) was significantly increased (p 0.005 ) to $200 \% \pm 31 \%$ compared with controls. Somatostatin was also measured and found to be unaltered $(110 \pm 6 \%)$ with respect to controls. Of the 19 patients studied 11 showed a consistent stimulatory effect on TRH content but it is unclear as yet why the effect is varied between patients.

These results may reflect a non-specific phenomenon related to MND serum. However, they are also consistent with the hypothesis that MND serum contains a factor(s) which is specifically activating TRH-producing cells in culture. Because of the probable trophic role of TRH on LMN function, characterisation of this factor(s) may be important in the understanding of MND.

\section{DEFECT OF PYRUVATE DEHYDROGENASE} COMPLEX IN INTERMITTENT ATAXIA

MA Birchin-Machin, LA Bindoff, D Gardner-Medwin, DM Turnbull. Royal Victoria Infirmary, Newcastle upon Tyne

Disturbances of pyruvate metabolism have been implicated in the aetiology of both Leigh's disease and familial ataxias. However, the precise enzyme diagnosis often remains uncertain. We report five males in one kindred who suffered from intermittent ataxia and the cerebral pathology in one case had some features of Leigh's disease. The pattern of inheritance strongly suggested $X$ linkage. Previous biochemical studies had shown slightly elevated blood lactate and pyruvate concentrations suggesting a defect in pyruvate metabolism. Recent further biochemical studies in skeletal muscle mitochondria from one of these patients revealed impaired oxidation of pyruvate $(38 \%$ of controls) and low pyruvate dehydrogenase (PDH) activity (50\% of controls). Immunoblot studies revealed a markedly low concentration of the Ela subunit of PDH with relatively normal concentrations of the other components. PDH complex is one of the major enzyme systems involved in the ccutrol of energy metabolism. The Ela subunit plays a key role in the function of the PDH complex as it contains both the active site and the main sites for regulation of overall PDH activity. The functional gene locus for the Ela subunit of human PDH complex has recently been localised by other workers to the X-chromosome. The deficiency of the Ela subunit explains the disturbance of pyruvate metabolism and the pattern of inheritance. The neurological abnormalities are presumably secondary to the consequent disturbance of energy metabolism.

\section{BOTULINUM TOXIN IN THE TREATMENT OF SPASTICITY}

TK Das, DM Park, AD Collings. Southend Hospital, Essex

Spasticity, once established, greatly complicates and interferes with the quality of movement. Even with vigorous physiotherapy it may lead to contractures. Medication or special techniques are seldom completely successful. We report treatment with botulinum toxin (B. Tox.) of eight patients (age 45-75 years) with spastic hemiplegia following stroke. Their spasticity was disabling and resistant to available therapy. B. Tox. diluted in normal saline was infiltrated into the biceps and common flexor muscles of the forearm at the motor point. The spasticity (assessed by the Oswestry scale) was substantially relieved and the range of joint movements (both at wrist and elbow) also improved considerably. Barthel scores also suggested improvement of the overall functional status. The injections were well tolerated and no side effect was noted.

Our observations indicate that local injections of B. Tox. may be extremely beneficial in relieving spasticity and improving functional ability. The effect of long term repeated administration remains to be evaluated.

\section{CT AND MR IMAGING IN TEMPORAL LOBE} EPILEPSY

R Duncan, D Hadley, P McPherson, J Patterson, I Bone. Southern General Hospital, Glasgow

Magnetic resonance imaging (MRI) is known to be superior to computed tomography (CT) in detecting lateralising abnormalities in temporal lobe epilepsy, but conventional $\mathrm{CT}$ is not best suited to the imaging of temporal lobe structures. We present a comparison of CT using modified temporal lobe cuts and MRI using a $0 \cdot 15 \mathrm{~T}$ resistive imager in 30 patients with intractable temporal lobe epilepsy.

CT detected abnormalities in 11 patients. In 10 this consisted of significant asymmetry of sylvian fissure or temporal horn. In one patient a small fleck of calcification was detected in the medial temporal lobe. MRI showed the calcified lesion as an area of gliosis or tumour. In total, MRI detected abnormalities in 15 patients. In eight this consisted of significant asymmetry of sylvian fissure or temporal horn. In two cases gliosis or tumour was detected in the medial temporal lobe: in one case there was also an increase in signal throughout the contralateral temporal lobe. In one case gliosis or tumour was detected in the temporal pole. In two cases there was an increase in signal throughout the whole of one temporal lobe. In one case an AVM or hamartoma was detected in the medial temporal lobe.

We conclude that MRI is better than CT in detecting lateralising abnormalities in temporal lobe epilepsy, even when the best CT technique is used.

TRANSIENT NEUROLOGICAL DYSFUNCTION IN ASSOCIATION WITH MALIGNANT MENINGITIS DG Grosset, I Draper, I Bone. Southern General Hospital, Glasgow

Episodes of unilateral transient third cranial nerve palsy with intermittent mono or hemiparesis occurred in two patients during severe recurrent headaches. Bradycardia and/or arterial hypertension was noted during the attacks. Malignant meningeal deposits and scar tumours of lung were found at post-mortem in both patients, who died suddenly during an attack. Transcranial Doppler sonography (TCD) performed dur- $\mathbb{D}$ ing a typical episode had shown very high systolic flow velocity, with diastolic falling to $\triangle$ zero, in one of these patients, with a return to normal values as the symptoms resolved. This suggests a vascular aetiology to these recurrent attacks; the mechanism may involve failure of release of neuropeptide vasodilators from the trigeminal nerve.

UNILATERAL ENCEPHALITIS IN THE VOGTKOYANAGA-HARADA SYNDROME

DG Grosset, PO Behan. Southern General Hospital, Glasgow

A 22 year old caucasian girl presented as a definite case of the Vogt-Koyanaga-Harada syndrome. There was a sub-acute meningoencephalitis culminating in a 36-hour period of coma. Depigmentation of skin, hair, and eyelashes preceded the neurological illness by six months, and progressed after recovery, which was full apart from some memory impairment. No ophthalmological involvement occurred prior to presentation or in a five month follow-up period. Electroencephalography (EEG) and single photon emission computed tomography (SPECT) during coma showed a predominantly unilateral encephalitis and cerebral hyperfusion, with return to normal at one week. Identifiable infective agents were excluded. The predominantly unilateral cerebral involvement in this case accords 
with the autopsy findings (the only such case known to the authors) in a similar case of the syndrome. Lateralisation of depigmentation, and vitiligo overlying organs involved in autoimmune disease, has been reported, and the pattern of cerebral involvement found adds weight to the autoimmune theory as an explanation for the encephalitis in this syndrome.

GUILLAIN-BARRÉ SYNDROME STEROID TRIAL Guillain-Barré Syndrome Steroid Trial Group. Guy's Hospital, London

This Medical Research Council funded trial is designed to demonstrate whether methylprednisolone will accelerate recovery from Guillain-Barré syndrome. Two hundred and forty eligible patients will be randomised to MP $500 \mathrm{mg}$ i-v, or placebo, daily for five days (120 in each group). Usual supportive treatment including plasma exchange is permitted. Additional steroid or immunosuppressive therapy may not be given for at least four weeks. Disability grade assessments on a scale ranging from $0=$ healthy to $6=$ dead, will be made by a "masked" assessor after two, four, 12, 14 and 48 weeks. The primary outcome criterion will be a 0.5 grade difference in disability grade change between treatment and placebo group after four weeks. With 100 patients in each group the trial has a $9.4 \%$ power of detecting such a difference at the $5 \%$ probability level (twotailed). Secondary outcome criteria will be a 0.5 grade difference in disability grade change after three months, reduction of duration of ventilation, and reduction of time to walk unaided. Sixty seven centres have agreed to participate in the trial. To date, 55 centres are ready to participate, 12 more centres have promised to make arrangements to participate, and 55 patients have entered the trial.

A PERIPHERAL MECHANISM FOR THE ATAXIA ASSOCIATED WITH THE MILLER FISHER SYNDROME OF ACUTE OPHTHALMOPLEGIA, ATAXIA AND AREFLEXIA

GA Jamal, M Donaghy. Southern General Hospital, Glasgow

It is not known whether the profound ataxia associated with the Miller Fisher syndrome (MFS) is due to dysfunction of the central or alternatively the peripheral nervous system. The relative preservation of sensation and muscle power has been cited as evidence for a cerebellar rather than peripheral nerve lesion despite the areflexia. Our study has investigated the alternative hypothesis that the ataxia reflects mismatching of peripheral inputs from muscle spindles and joint position receptors to the cerebellum. In four patients with MFS, proprioceptive silent periods were recorded serially throughout the illness and correlated with joint position sensation and clinical severity of the ataxia. All patients displayed consistent mismatching between joint receptor and proprioceptive input at times when their ataxia was severe. Mismatching gradually resolved as the ataxia recovered. We conclude that ataxia in MFS can be adequately explained by peripheral nerve dysfunction without invoking a lesion of the central nervous system.

\section{A LONGITUDINAL STUDY OF SENSORY}

CHANGES IN THE GUILLAIN-BARRÉ SYNDROME GA Jamal, JP Ballantyne. Southern General Hospital, Glasgow

The aetiology, incidence, characteristics and outcome of the sensory disturbances in patients with the Guillain-Barré syndrome (GBS) are unclear. In particular information on the longitudinal course of these sensory disturbances and concomitant involvement of small fibre population is limited. We performed serial examinations on 20 patients with GBS from onset for 18 months with clinical assessment, sensory conduction and quantitative thermal vibration studies. Twelve patients presented with sensory disturbances and these developed in four more within the first week. Sensory signs were present in 13 patients. Abnormality of one or more of the sensory action potentials (SAPs) was found in 18 patients at one month from onset. Median and ulnar SAPs were more frequently abnormal than the sural SAP. Thermal thresholds were abnormal within one month in 18 patients more frequently at the ankle. Vibration perception threshold (VPT) was abnormal by one month after onset in 19 patients. Eighteen patients had no sensory symptoms by 18 months. At this time all 18 patients had normal thermal thresholds while 15 had normal VPT. Two patients with a substantial residual disability at 18 months continued to show evidence of clinical sensory disturbances and in these both thermal and VPT remained abnormal. Neurophysiological signs of sensory disturbance in both large and small nerve fibre populations are common in patients with GBS though less frequent and less prominent than motor disturbances. Quantitative investigation by thermal and VPT estimation yields a higher percentage of abnormality.

\section{FURTHER EVIDENCE FOR THE SPECIFICITY OF}

TRIPLE T: PREDOMINANT SMALL FIBRE

NEUROPATHY AND FRIEDREICH'S ATAXIA COMPARED

GA Jamal, S Hansen, JP Ballantyne. Southern General Hospital, Glasgow

Selective loss of peripheral large diameter nerve fibres is known to occur in patie with Friedreich's ataxia (FA) on the basisef both histological and in vitro elect physiological studies of the sural nerFe. Small diameter nerve fibres are preservedat least early in the course of the diseags. Fourteen patients with FA aged 17-37 ye每s were examined with the Thermal Thresho Technique (TTT) to determine the relative specificity of the method as a test for the functional integrity of the small nerve fibite population. The results were compared wfth a matched control group. All 14 patients had abnormal conventional sensory conducti $\overline{\bar{B}}$ studies and elevated vibration thresholds. $\overline{\Phi_{n}}$ 12 of the 14 patients normal thermal thicsholds were found at both ankles and wrists. Two patients with clinically advanced d\&sease had raised thermal thresholds at te ankle alone. Both patients had abnornial sensation to pinprick on clinical examin $\overrightarrow{3}$ tion. In a previous study we have demog strated an abnormality of thermal thresho-fis in all 25 patients with predominant small fibre neuropathy using TTT. By contrast, 骓 FA, a condition associated with large fibre involvement the technique has demonstrated, as anticipated, a relative pres tion of the small fibre population. The TIF is a valuable tool in the separation of tbe large from the small fibre neuropathies

AN ORTHODROMIC STUDY OF DIGITAL SENSORY NERVES IN CARPAL TUNNEL SYNDROME

RAL Macdonell, M Schwartz, M S The London Hospital, London

The most sensitive means of establishing diagnosis of carpal tunnel syndrome (CTS, is through analysis of median nerve sensory conduction at the wrist. This is commonily performed by stimulating two digital braf ches of the median nerve using ring ele trodes around a digit. The digit most likely $\overrightarrow{\text { हु }}$ show abnormalities in CTS is controversia

Thirty-six females ( 56 hands) with clinical and electrophysiological evidence off idiopathic carpal tunnel syndrome were studied. Each digital branch of the median nerve was stimulated and the sensory nerwe action potential (SNAP) was recorded at the wrist. The threshold for stimulus detection; amplitude, latency and conduction velocifo of the SNAP were compared with those found for the corresponding digital nerve in a group of age and sex matched controls. The control subjects had no clinical or electro physiological evidence of CTS.

The most frequent abnormality (93\%) in the CTS patients was either a small amplitude or absent SNAP followid stimulation of the lateral side of the ritg finger. The same abnormality was foupg from the index finger in $77 \%$ and from the 
middle finger in $84 \%$. These findings suggest that the ring finger is the most suitable for sensory nerve conduction study in possible CTS.

\section{EVER YDAY MEMORY IN MULTIPLE SCLEROSIS - A POPULATION STUDY}

S McIntosh-Michaelis, S Wilkinson, $\mathbf{M}$ Roberts, I Diamond, D McLellan, J Martin. Southampton General Hospital, Southampton

A one in two sample $(n=200)$ stratified by age, disease duration and disability was constructed from a population based prevalence register of 411 people with multiple sclerosis (MS). One hundred and forty seven $(74 \%)$ of the sample were interviewed. They were representative with respect to age, duration of disease and disability. The mean age was 48 years, median disease duration 13 years, and the median Kurtzke Expanded Disability Status Scale rating 6.0. A control group of 34 people with rheumatoid arthritis was also interviewed. The Rivermead Behavioural Memory Test and two tests of frontal lobe function (The Controlled Oral Word Association and Modified Wisconsin Card sorting Test) were administered to both groups as part of a fuller psychometric assessment. Forty nine $(34 \%)$ people with MS had scores of six or less on the Rivermead Behavioural Memory Test compared with four $(12 \%)$ of the controls $\left(\chi^{2} 5.3 \mathrm{sig}<\right.$ $0.05 \mathrm{DF}=1)$. Log-linear modeling of the memory scores showed significant relationships between poor memory function, duration of disease over 10 years and Kurtzke Expanded Disability Status Scores over seven. The use of baclofen or benzodiazepines was not a significant explanatory variable in this model. Forty five $(33 \%)$ of those people completing the tests had scores in the clinically abnormal range for one or both of the two tests of frontal lobe function.

\section{MEDIAN CORTICAL TRANSIT TIME IN} DIFFERENT TYPES OF ACUTE CEREBRAL INFARCTION

AR Naylor, MV Merrick, RJ Sellar, PAG Sandercock, CP Warlow. Western General Hospital, Edinburgh

Sixteen patients with first ever stroke had serial clinical evaluation, CT scanning, extra and transcranial Doppler studies and quantification of regional median cortical transient times (MCTT), which gives an indirect measure of cerebral perfusion reserve. The aim of the study was to examine MCTT abnormalities in different types of cerebral infarction. In seven of seven patients with total anterior circulation infarction (TACI), MCTT abnormalities were concordant with the clinical features, CT and
Doppler findings. In two of four patients with partial anterior circulation infarction (PACI), there was discordance between MCTT and CT findings. Two patients with lacunar infarction (LACI) had normal MCTT and Doppler studies.

There was therefore concordance between MCTT, clinical, CT and Doppler abnormalities in patients with TACI and LACI. The discordance between CT and MCTT in two patients with PACI is of interest. MCTT is simple and cheap to measure and these results suggest that it may be of considerable value in the study of the pathophysiology of acute ischaemic stroke.

\section{POSTURAL CONTROL AND THE EFFECT OF} VISUAL FEEDBACK IN PARKINSON'S DISEASE JA Waterston, MB Hawken, S Tanyeri, C Kennard. The London Hospital, London

Postural instability is a major clinical problem in Parkinson's disease. Fore-aft postural sway was examined in 14 male Parkinsonian patients and 14 age matched controls, under various conditions. The supporting platform was used in one of two conditions; either being fixed, or moved, in response to sway, thereby diminishing footankle proprioceptive input. In addition, three visual conditions were provided. Firstly sway was measured with absent visual input (eyes closed). In the other two conditions the subjects either viewed a stationary target (eyes open) or a target which moved in response to the sway signal input (visual feedback).

There was a suggestion of impaired performance under all conditions in the Parkinsonian group, the result only just reaching statistical significance $(p<0.03)$. Both groups showed increasing sway with the removal of visual or ankle proprioceptive feedback ( $p<0.001$ ). When additional visual feedback was provided, both groups were able to improve their performance over the "eyes open" condition ( $p<0.001)$. This result suggests that Parkinsonian subjects can significantly improve their motor performance with the use of appropriate visual feedback. A clinical correlate exists in the use of sensory tricks in Parkinson's disease to overcome functional disabilities. Therapeutic options may be possible with visually guided motor retraining.

MONONEUROPATHY AND MULTIPLE MONONEUROPATHY AS THE PRESENTATION OF EPITHELIOID SARCOMA-A CAUTIONARY TALE AI Weir, $R$ Reid. Southern General Hospital, Glasgow

A 20 year old lady presented with a complete radial nerve palsy one month after a fall. This was assumed to be traumatic but did not recover. Six years later she was re-referred with a new complaint of hand paraesthesiae and numbness. Clinical and neurophysiological examinations confirmed a bilateral ulnar entrapment neuropathy at the elbow and decompression was performed on the symptomatic side. Following this, progressive weakness of forearm muscles was noted. Re-examination revealed a point of severe tenderness with a Tinel's sign over the brachial plexus in the axilla. Exploratory surgery demonstrated a striking constricture around the entire brachial plexus, and biopsy confirmed an epithelioid sarcoma.

A 33 year old lady presented with a three year history of paraesthesiae and weakness in the median nerve territory associated with mechanical limitation of elbow extension. Clinical and neurophysiological examination demonstrated a lesion of the main trunk of the median nerve at or just proximal to the elbow. Exploratory surgery revealed a constriction around the brachial artery and median nerve at the elbow and biopsy confirmed an epithelioid sarcoma.

In both cases radical excision with amputation was performed and there is noक evidence of recurrence to date.

SEASONAL AND DIURNAL VARIATION IN STROKE ONSET: THE OXFORDSHIRE COMMUNITY STROKE PROJECT

SJ Wroe, PAG Sandercock, J Bamford M Dennis, J Burn, CP Warlow, J Slattery, L Jones. Western General Hospital, Edinburgh

We examined data on seasonal and diurnal variation in the incidence of first-ever stroke and on activity at the time of stroke onset in 675 patients in the Oxfordshire Community Stroke Project. There were 514 cases of definite cerebral infarction (CI), 60 cases of definite intracerebral haemorrhage (ICH), and 32 cases of definite subarachnoid haemorrhage (SAH). The pathological type of stroke was not known in 69 cases. There was a significant excess of $\mathrm{ICH}$ and of SAH in winter (December to February, $p<0.01$ ). There is no such seasonal difference in the incidence of $\mathrm{CI}$ although slightly more infarcts occurred in summer. For all types of stroke, time of onset was most often between 06.00 and 12.00 hours ( $49 \%$ of $\mathrm{CI}, 41 \%$ of ICH, $43 \%$ of SAH) and most occurred whilst awake. SAH but not CI or ICH was associated with moderate or strenuous activity at $N$ the time of stroke onset. Our results are more reliable than earlier studies based on stroke $N$ mortality statistics and hospital admission N data which may be biased and provide only indirect evidence of changes in stroke incidence. 